\title{
The Equilibrium State of Colliding Electron Beams *
}

\author{
R.L. Warnock, Stanford Linear Accelerator Center \\ Stanford University, Stanford, CA 94309 \\ J.A. Ellison, Department of Mathematics and Statistics \\ University of New Mexico, Albuquerque, NM 87131
}

December 12, 2003

\begin{abstract}
We study a nonlinear integral equation that is a necessary condition on the equilibrium phase space distribution function of stored, colliding electron beams. It is analogous to the Haïssinski equation, being derived from Vlasov-Fokker-Planck theory, but is quite different in form. The equation is analyzed for the case of the Chao-Ruth model of the beam-beam interaction in one degree of freedom, a so-called strong-strong model with nonlinear beam-beam force. We prove existence of a unique solution, for sufficiently small beam current, by an application of the implicit function theorem. We have not yet proved that this solution is positive, as would be required to establish existence of an equilbrium. There is, however, numerical evidence of a positive solution. We expect that our analysis can be extended to more realistic models.
\end{abstract}

\section{Introduction}

In the theory of stability of stored beams a primary step should be the study of equilibrium states, expected to exist at low current. An equilibrium state should become unstable at some threshold in current, but in order to compute the threshold we must linearize the kinetic equation (Vlasov or Vlasov-Fokker-Planck ) about the equilibrium phase space distribution. Historically, investigators have often linearized the Vlasov equation about some state that might be at best a rough approximation to an equilibrium. This may be excused by the fact that determination of the equilibrium is an infinite-dimensional nonlinear problem, in general rather difficult.

There is one case in which there is a widely known theory of equilibrium, which makes some contact with experiment; namely, the case of longitudinal motion of a single bunched electron beam in a storage ring, subject to a wake field [1,2]. The theory is based on a model in which the exact longitudinal wake field is replaced by its average over one turn. The

*Work supported in part by Department of Energy contracts DE-FG03-99ER41104 and DE-AC03$76 \mathrm{SF} 00515$. 
averaged wake of course depends only on the distance between source and test particles, not on the position in the ring. With such a wake one may seek a time-independent, factorized solution of the Vlasov-Fokker-Planck (VFP) equation; namely, a product of a Gaussian in the canonical momentum $p$ (proportional to the energy deviation) and the charge density $\rho(q)$, where $q$ is the canonical coordinate (proportional to the distance from the synchronous particle). The equation is satisfied by such a factorized form, provided that the charge density satisfies the Haïssinski equation [1,2], a nonlinear integral equation. If the wake field satisfies a mild restriction, it is not difficult to prove that the equation has a unique solution in a large function space $\mathcal{S}$, at sufficiently small current [2]. The corresponding solution of the VFP equation is the unique, small-current solution satisfying the principle of detailed balance ( with $\rho \in \mathcal{S}$ ).

There are many ways in which this prototype theory of equilibrium might be extended. For instance, one might include multi-bunch beams, long-range wakes from cavity resonators or resistive walls, nonlinear r.f. buckets, proton beams with non-Gaussian distribution in $p$, localized wakes not averaged over azimuth. A generalization to $2 d$-dimensional phase space might also be made, and in fact Dressler [3] has already done that for $d \geq 3$ in a mathematical study of VFP equilibria in the context of plasma physics. He has an external confining potential, analogous to an r.f. bucket, and a Vlasov coherent force derived from a Coulomb potential or mollified potential (smooth and bounded). The analysis gives results on existence and uniqueness of equilibria, using factorization and an integral equation on $d$ dimensional configuration space, quite in analogy to Haïssinski theory. Another possible line of generalization would be to allow "colored" noise, in which the autocorrelation function is not a delta function [4].

Here we are interested in two counter-rotating beams in collision. In mathematical aspects the problem has similarities to the case of a single beam with localized wake contributions. The beam-beam collision gives a large transverse force that substantially modifies the beams at every collision. Consequently, the equilibrium state, if any, cannot be timeindependent. Rather, it must be defined as a phase space distribution that is periodic in azimuthal position $s$. The same may be said of the exact equilibrium in any storage ring, with or without beam-beam collisions. The Haïssinski solution is time-independent only because it is based on the averaged wake field. As a zeroth approximation one could also do averaging in the beam-beam problem, distributing the localized beam-beam kick over a full turn. This has been done in linear stability studies [5]. Here we wish to avoid such a step, accounting fully for the localization. It that case the distribution cannot be factorized. We must expect the equilibrium equation to be an integral equation for functions on phase space, not just on configuration space as in the Haïssinski and Dressler theories. We derive and analyze the simplest instance of such an equation, retaining the full nonlinearity of the beam-beam force.

The integral equation can be viewed as a fixed point problem in a function space. One can try to apply the usual fixed point theorems of nonlinear functional analysis, the contraction mapping theorem and Schauder's theorem [6], to prove existence of a solution. We had no initial success with that approach, and turned instead to the implicit function theorem in Banach space. The solution for zero beam current is known, and the implicit function 
theorem allows one to show that there is a locally unique continuation to non-zero current. Although the operator of the problem in fixed point form preserves positivity of a probability distribution to which it is applied, we have not been able to show that the solution from the implicit function argument is positive. Consequently, our proof of the existence of an equilibrium is not complete, contrary to our claim in a preliminary report [7].

In retrospect we find that the integral equation can be recast as a fixed point problem with contractive operator, but only in terms of an auxiliary operator that appears in the present analysis; namely, the operator $G_{f}\left(f_{0}, 0\right)^{-1}$ treated in Section 3.3. Since a proof based on the contractive formulation does not lead to stronger results, and only rearranges the argument, we defer an exposition of that and other formulations to a later report.

Some background to the present study is found in a recent paper [8]. There we gave a derivation of the beam-beam force and made an analytic study of equilibria by linearizing the force, but retaining the quadratic nonlinearity of the Vlasov equation. We also carried out a numerical integration of the fully nonlinear VFP system. Here we adopt the notation and equations of motion as given in Ref. [8].

\section{Formulation of the Problem}

We employ definitions of accelerator physics as treated in texts such as Refs.[9] and [10]. We treat vertical transverse motion with normalized phase-space variables $(q, p)$ defined in terms of the lattice function $\beta(s)$ and emittance $\epsilon$ as

$$
q=y(\beta(s) \epsilon)^{-1 / 2}, \quad p=\left(\beta(s) y^{\prime}-\beta^{\prime}(s) y / 2\right)(\beta(s) \epsilon)^{-1 / 2},
$$

where $y$ is the vertical displacement and the prime denotes $d / d s$. The Hamiltonian of motion unperturbed by the beam-beam interaction is $H=\left(p^{2}+q^{2}\right) / 2$ and the independent "time" variable of Hamilton's equations is the phase advance $\theta=\int_{0}^{s} d u / \beta(u)$. We define the emittance as the expected value of the Courant-Snyder invariant $J$ in the unperturbed equilibrium state; see Appendix A. We distinguish the two beams by a subscript $i=1,2$. The parameters $\beta, \epsilon$ in (1) depend on $i$, so that the coordinates and time are defined differently for the two beams. Which definition is being used will either be clear from the context, or will be recognized explicitly when necessary. When the theory is generalized to allow more than one degree of freedom, the phase advance is not a suitable independent variable. There is no difficulty in using $s$ in place of $\theta$, as is well known.

Between beam-beam collisions the phase-space distribution function for beam $i$, denoted by $f_{i}(q, p, \theta)$, propagates according to the Fokker-Planck equation,

$$
\frac{\partial f_{i}}{\partial \theta}+p \frac{\partial f_{i}}{\partial q}-q \frac{\partial f_{i}}{\partial p}=2 \alpha_{i} \frac{\partial}{\partial p}\left[p f_{i}+\frac{\partial f_{i}}{\partial p}\right] .
$$

If the left hand side were set to zero, we would have the Liouville equation for an harmonic oscillator, the oscillations being motion transverse to the beam direction in fields of focusing magnets. The right hand side is the Fokker-Planck term accounting for damping and diffusion 
due to synchrotron radiation in quanta. The damping constant is

$$
\alpha_{i}=\frac{1}{2 \pi \nu_{i}\left(n_{d}\right)_{i}},
$$

a small dimensionless parameter that typically could be around $10^{-4}$ or less. The number of turns in a damping time is $n_{d}$ and $2 \pi \nu$ is the change in $\theta$ per turn. Our dynamical variables are scaled so that the diffusion constant, the coefficient of $\partial^{2} f_{i} / \partial p^{2}$, is equal to $2 \alpha_{i}$. Such a scaling is possible because the right hand side of (2) is not homogeneous in $p$.

At the point where the two beams are brought into collision (called the interaction point or IP) the distribution function changes discontinuously. This happens at $\theta=2 \pi n \nu_{i}$ for beam $i$, with $n=0,1,2, \cdots$. The change of the distribution is so as to preserve probability and has the form

$$
f_{i}\left(q, p, 2 \pi n \nu_{i}+0\right)=f_{i}\left(q, p-F_{j}(q, n), 2 \pi n \nu_{i}-0\right), \quad j \neq i,
$$

where $F_{j}(q, n)$ is the normalized momentum imparted to a particle at location $q$ in beam $i$ by beam $j$ at the instantaneous collision. The -0 and +0 indicate instants just before and after the collision, respectively. The right hand side of (4) is the result of applying the Perron-Frobenius operator [2] for the beam-beam kick map to the function $f_{i}\left(q, p, 2 \pi n \nu_{i}-0\right)$.

The Chao-Ruth model $[11,8]$ is intended to represent flat beams, with large $x: y$ aspect ratio. The force on a particle in beam $i$ is approximated as though it came from infinite uniform planes of charge perpendicular to the $y$-axis, distributed with a density $\rho_{j}(y)$ characterizing the other beam $j$. This force is concentrated in time, however, at the instant of collision. In this model the momentum kick to beam $i$ at the $n$-th collision caused by beam $j$, also called the collective force, has the form

$$
F_{j}(q, n)=-(2 \pi)^{3 / 2} \xi_{j} \int \operatorname{sgn}\left(r_{i j} q-q^{\prime}\right) f_{j}\left(q^{\prime}, p^{\prime}, 2 \pi n \nu_{j}-0\right) d q^{\prime} d p^{\prime}, \quad i \neq j
$$

The signum function $\operatorname{sgn}(x)$ is 1 for $x>0$ and -1 for $x<0$. The integration of $f_{j}$ over $p^{\prime}$ gives the charge density of beam $j$. Here $q$ refers to beam $i$ and $q^{\prime}$ to beam $j$, and the parameter $r_{i j}=\left(\beta_{i}^{*} \epsilon_{i} / \beta_{j}^{*} \epsilon_{j}\right)^{1 / 2}$ accounts for the difference in the definition of the two. The asterisk specifies that the beta function be evaluated at the interaction point. The dimensionless beam-beam parameter $\xi_{j}$ is proportional to the current in beam $j$, but depends on the kinematic parameters of beam $i$; it is defined as

$$
\xi_{j}=\frac{r_{e}}{(2 \pi)^{1 / 2}}\left(\frac{\beta^{*}}{\gamma \sigma_{y}}\right)_{i} n_{j}, \quad i \neq j .
$$

Here $n=N / L_{x}$ is the number of particles per unit length in the $x$-direction; i.e., $N$ is the number of particles and $L_{x}$ is the bunch width. Also, $r_{e}=e^{2} /\left(4 \pi \epsilon_{0} m c^{2}\right)$ is the classical electron radius, $\gamma$ is the Lorentz factor, and $\sigma_{y}=\left(\beta^{*} \epsilon\right)^{1 / 2}$ is the bunch height.

For propagation of the distribution function between IP kicks, we have in (2) a linear Fokker-Planck equation with harmonic force. The propagator or fundamental solution of 
that equation is known [12], namely a function $\Phi\left(z, z^{\prime}, \theta\right), z=\left(z_{1}, z_{2}\right)=(q, p)$ such that for any initial distribution $f(z, 0)$ the solution at time $\theta$ is

$$
f(z, \theta)=\int \Phi\left(z, z^{\prime}, \theta\right) f\left(z^{\prime}, 0\right) d z^{\prime}
$$

where $d z^{\prime}=d q^{\prime} d p^{\prime}$. The propagator depends of course on the beam index $i$, but we suppress that index whenever we are concerned with just one beam at a time. There are several equivalent representations of $\Phi$. The following form, derived from a probabilistic argument in Appendix A, is especially appealing and convenient for the present work:

$$
\begin{aligned}
\Phi\left(z, z^{\prime}, \theta\right)=\quad & \frac{1}{2 \pi(\operatorname{det} \Sigma(\theta))^{1 / 2}} \exp \left[-\left(z-e^{A \theta} z^{\prime}\right)^{T} \Sigma(\theta)^{-1}\left(z-e^{A \theta} z^{\prime}\right) / 2\right] \\
& \Sigma(\theta)=I-e^{A \theta} e^{A^{T} \theta} .
\end{aligned}
$$

Here $T$ denotes transposition and $e^{A \theta}$ is the transfer matrix (principal solution matrix) for the single-particle harmonic motion with damping. With damping constant $\alpha$ we have

$$
e^{A \theta}=e^{-\alpha \theta} R(\theta)
$$

where

$$
R(\theta)=\left(\begin{array}{cc}
\cos \Omega \theta+(\alpha / \Omega) \sin \Omega \theta & (1 / \Omega) \sin \Omega \theta \\
-(1 / \Omega) \sin \Omega \theta & \cos \Omega \theta-(\alpha / \Omega) \sin \Omega \theta
\end{array}\right),
$$

In Appendix $\mathrm{B}$ we show that $\Sigma$ is positive definite for $\theta>0$. Also, $\Sigma$ tends to the unit matrix at large $\theta$, according to (8), (9), and (10). For small $\alpha$ the matrix $R$ is close to a rotation, and $\Sigma$ has the form

$$
\Sigma(\theta)=2 \alpha\left(\begin{array}{cc}
\theta-\cos \theta \sin \theta & -\sin ^{2} \theta \\
-\sin ^{2} \theta & \theta+\cos \theta \sin \theta
\end{array}\right)+\mathcal{O}\left(\alpha^{2}\right)
$$

Even though the realistic value of $\alpha$ is small our analysis requires only $0<\alpha<1$, which is to say that the harmonic oscillator is not over-damped.

Let $\boldsymbol{\Phi}$ denote the operator corresponding to the kernel $\Phi\left(z, z^{\prime}, \theta\right)$ in $(7)$. The action of $\boldsymbol{\Phi}$ has a simple expression in Fourier space. Writing $\hat{h}$ for the Fourier transform of $h$, we have

$$
\widehat{\Phi h}(v)=\exp \left[-v^{T} e^{A \theta} \Sigma e^{A^{T} \theta} v / 2\right] \hat{h}\left(e^{A^{T} \theta} v\right) .
$$

There is an interesting alternative formulation of our problem based on this formula, which we hope to explore in a later communication.

We can now set down a system of integral equations for the equilibrium distribution. The equations are for the distributions evaluated just after the IP, $f_{i}(z, 0+)$. Henceforth we suppress the time specification $0+$. Starting with $f=\left(f_{1}, f_{2}\right)$, we propagate one turn by (7) with $\theta=2 \pi \nu$, and then apply the beam-beam kicks according to (5). For equilibrium 
(periodicity), the result must be the starting $f$. To state this in equations we first define the linear operator $\mathbf{L}_{j}$ by

$$
\begin{aligned}
& \mathbf{L}_{j} f(q)=(2 \pi)^{3 / 2} \int \operatorname{sgn}\left(r_{i j} q-q^{\prime}\right) \mathbf{K}_{j} f\left(z^{\prime}\right) d z^{\prime}, i \neq j, \\
& \mathbf{K}_{j} f(z)=\int K_{j}\left(z \mid z^{\prime}\right) f_{j}\left(z^{\prime}\right) d z^{\prime},
\end{aligned}
$$

where $K_{j}$ is the Fokker-Planck propagator for one turn,

$$
K_{j}\left(z \mid z^{\prime}\right)=\Phi_{j}\left(z, z^{\prime}, 2 \pi \nu_{j}\right) .
$$

The integral equations take the form

$$
f_{i}(z)=\mathbf{K}_{i} f\left(q, p+\xi_{j} \mathbf{L}_{j} f(q)\right), \quad i, j=1,2, \quad i \neq j,
$$

with

$$
\int f_{i}(z) d z=1
$$

It is essential that the normalization constraint (18) be regarded as part of the definition of the mathematical system; otherwise in Eqs.(17) there is nothing to set the scale of the beam-beam force. We choose to build in the constraint by redefining the integral equation, multiplying the left hand side of (17) by $\int f_{i}(\zeta) d \zeta$. Then, since $\int K_{i}\left(z \mid z^{\prime}\right) d z=1$, any solution of the modified equation will automatically satisfy $\left(\int f_{i}(z) d z\right)^{2}=\int f_{i}(z) d z$. Since the implicit function theorem will rule out $\int f_{i}(z) d z=0$, we shall then be assured that our solution satisfies (18). Thus our task is to analyze the pair of equations

$$
G(f, \xi)=0
$$

where $G=\left(G_{1}, G_{2}\right), \xi=\left(\xi_{1}, \xi_{2}\right)$ with

$$
G_{i}(f, \xi)(z)=f_{i}(z) \int f_{i}\left(z^{\prime}\right) d z^{\prime}-\mathbf{K}_{i} f\left(q, p+\xi_{j} \mathbf{L}_{j} f(q)\right), \quad i \neq j .
$$

In the special case of beams with identical parameters, the two equations in (19) have the same form. Then if $\left(f_{1}, f_{2}\right)$ is a solution, so must be $\left(f_{2}, f_{1}\right)$. Our analysis will show that the small-current solution is unique, so for it $f_{1}=f_{2}$. Consequently, for the case of identical beams at small current it suffices to solve a single equation $G(f, \xi)=0$, where

$$
G(f, \xi)(z)=f(z) \int f\left(z^{\prime}\right) d z^{\prime}-\int K\left(q, p+\xi \mathbf{L} f(q) \mid z^{\prime}\right) f\left(z^{\prime}\right) d z^{\prime}=0 .
$$

with

$$
\mathbf{L} f(q)=(2 \pi)^{3 / 2} \iint \operatorname{sgn}\left(q-q^{\prime}\right) K\left(z^{\prime} \mid z^{\prime \prime}\right) f\left(z^{\prime \prime}\right) d z^{\prime} d z^{\prime \prime} .
$$

To avoid notational clutter we present our proof for the identical beam problem (21). It will be obvious that the proof goes through in essentially the same way for the general problem (19). 
We know the solution of $(21)$ at $\xi=0$; it is the Gaussian equilibrium in the absence of beam-beam force,

$$
G\left(f_{0}, 0\right)=0, \quad f_{0}=\frac{1}{2 \pi} \exp \left(-\frac{1}{2}\left(q^{2}+p^{2}\right)\right) .
$$

We apply the implicit function theorem in a Banach space to prove that this solution can be continued in a unique way to a solution $f(\xi)$ of $(21)$ for small $\xi \neq 0$. Let us first recall the intuitive basis of the theorem in finitely many dimensions, so that (21) represents $n$ real (generally nonlinear) equations in $n$ unknowns $f_{j}, j=1, \cdots, n$. We wish to solve for the $f_{j}$ as a function of the parameter $\xi$, supposing that a solution $f_{0 j}$ for $\xi=0$ is known. Supposing that $G$ is smooth, we can expand it by Taylor's formula with remainder $R$ about the point $\left(f_{0}, 0\right)$ :

$$
G(f, \xi)=G_{f}\left(f_{0}, 0\right)\left(f-f_{0}\right)+G_{\xi}\left(f_{0}, 0\right) \xi+R(f, \xi)=0 .
$$

If the Jacobian matrix $G_{f}=\left\{\partial G_{i} / \partial f_{j}\right\}$ is non-singular at the expansion point, and the nonlinear remainder $R$ is small, an approximate solution of our problem is

$$
f(\xi) \approx f_{0}-G_{f}\left(f_{0}, 0\right)^{-1} G_{\xi}\left(f_{0}, 0\right) \xi .
$$

The implicit function theorem takes into account the nonlinear term, and assures us that for sufficiently small $\xi$ there will be a unique exact solution of (21) close to the approximation (25).

For our infinite-dimensional case we look for a solution in a certain set of continuous functions that form a Banach space. A Banach space is a normed linear space that is complete in the topology provided by the norm. Completeness means that every Cauchy sequence $\left\{f_{n}\right\}$ has a limit in the space: if for any $\epsilon>0$ we have $\left\|f_{n}-f_{m}\right\|<\epsilon$ for $n, m>N(\epsilon)$, then there is an $f$ so that $\left\|f-f_{n}\right\| \rightarrow 0, n \rightarrow \infty$.

For an interesting history and overview of implicit function theorems see [13]. We apply the following theorem, which is not the most general but adequate for our purpose [14]:

Theorem: Let $B$ be a Banach space, and suppose that $G(f, \xi)$ maps $B \times I$ into $B$, where $I=(-\Delta \xi, \Delta \xi)$ is an open interval, the domain of $\xi$. Suppose also that $G$ has (Fréchet) partial derivatives $G_{f}(f, \xi), G_{\xi}(f, \xi)$, continuous in $B \times I$ (equivalently, $G$ is continuously differentiable on $B \times I$ ). Let $f_{0} \in B$ be a solution of $G\left(f_{0}, 0\right)=0$, and suppose that $G_{f}\left(f_{0}, 0\right)$ is a bounded linear map of $B$ onto $B$ with a bounded inverse. Then there exists a unique solution $f(\xi)$ of $G(f, \xi)=0$ such that $f(0)=f_{0}$, for $\xi$ in some interval $I_{0}=(-\delta \xi, \delta \xi) \subset$ $I, \quad \delta \xi \neq 0$. Moreover, for $\xi \in I_{0}$ this solution has a continuous derivative with respect to $\xi$ and $\left(G_{f}(f(\xi), \xi)\right)^{-1}$ exists. The derivative is given by $f^{\prime}(\xi)=$ $-\left(G_{f}(f(\xi), \xi)\right)^{-1} G_{\xi}(f(\xi), \xi)$.

The partial derivative $G_{f}(f, \xi): B \rightarrow B$ is a bounded linear operator such that

$$
\lim _{\|h\| \rightarrow 0} \frac{1}{\|h\|}\left\|G(f+h, \xi)-G(f, \xi)-G_{f}(f, \xi) h\right\|=0 .
$$

Continuity on $B \times I$ means that for any $\epsilon>0$ there is a $\delta$ such that

$$
\left\|G_{f}\left(f_{1}, \xi_{1}\right)-G_{f}\left(f_{2}, \xi_{2}\right)\right\|<\epsilon,
$$


when $\max \left(\left\|f_{1}-f_{2}\right\|,\left|\xi_{1}-\xi_{2}\right|\right)<\delta$. Here the operator norm is

$$
\left\|G_{f}(f, \xi)\right\|=\sup _{\|h\|=1}\left\|G_{f}(f, \xi) h\right\|,
$$

where sup means least upper bound. The derivative $G_{\xi}(f, \xi): I \rightarrow B$ is defined similarly. In our case, $G_{f}$ will be an integral operator and $G_{\xi}$ will be multiplication by a function.

The theorem alone does not give us an estimate of the size of the interval $I_{0}$ in which the solution exists. In specific cases analytic estimates can be made, but they may be pessimistic. In our problem, we mainly seek assurance that an equilibrium exists for sufficiently small current. We shall have to rely on numerical calculations to determine a maximum interval of existence. Calling on experience with the Haïssinski equilibrium, we expect that the equilibrium will be stable at small current, and with increasing current will continue to exist long after it becomes unstable.

As is usual in applications of functional analysis, some experimentation is required to find a suitable space $B$. The space must be designed to match the properties of the problem, and there is no expectation that the choice will be obvious or unique. Physicists are most familiar with Hilbert space (an example of Banach space) and its $L^{2}$ norm, but squaring and integrating to compute the norm is often quite awkward, especially in nonlinear problems. It is usually easier to follow the model of a simple example of a Banach space, namely the set of all continuous functions $f$ on the closed interval $[0,1]$, with the norm $\|f\|=\sup _{x \in[0,1]}|f(x)|$, where sup (supremum) means "least upper bound". One can extend the domain of the functions to the real line $\mathbb{R}$ and also ensure appropriate decay of the functions at infinity by including a weight function $w(x)$ in the norm, thus $\|f\|=\sup _{x \in \mathbb{R}} w(x)|f(x)|$, where $w$ is a positive function that grows at large $x$. The choice of $w$ will depend on specific traits of the operator being analyzed. In some problems it may be necessary to have terms in the norm that involve derivatives or other information about the function; fortunately none are needed in the present case.

After various estimates of integrals we find that a suitable $B$ consists of all continuous functions $f$ on the phase space $\mathbb{R}^{2}$ such that the following expression, identified as the norm, is finite:

$$
\|f\|=\sup _{z \in \mathbb{R}^{2}}|w(z) f(z)|, \quad w(z)=1+|z|^{2 a}, \quad a>2 .
$$

We suppress reference to the dependence of $B$ on the fixed number $a$. In Appendix $\mathrm{C}$ we show that the linear space of continuous functions $f$ with $\|f\|<\infty$ is complete in the metric $d(f, g)=\|f-g\|$. The choice of a weight function with power behavior is convenient, but other functions with monotonic behavior in $|z|$ could work as well. We are led in stages to the requirement $a>2$. The first part of the proof, in Sections 3.1 and 3.2, requires only $a>1$. For the last step of the proof, proving convergence of the series that defines $G_{f}\left(f_{0}, 0\right)^{-1}$, we have to assume $a>2$.

From a physical viewpoint this $B$ is a very "big" space, since it contains some functions with polynomial decay at infinity, whereas numerical and experimental results suggest that the solution actually decays much more rapidly, somewhat as a Gaussian. Our existence proof of a unique solution works for any $a>2$, but the requirement on smallness of the beam current parameter $\xi$ may become more strict as $a$ is increased. The choice of a small 
$a$, barely bigger than 2, has mathematical appeal in the assertion of uniqueness in a broad universe.

For the case of dissimilar beams the Banach space would consist of pairs $\left(f_{1}, f_{2}\right)$ of continuous functions, and the norm could be

$$
\|f\|=\max _{i} \sup _{z \in \mathbb{R}^{2}}\left|\left(1+|z|^{2 a}\right) f_{i}(z)\right|, \quad a>2 .
$$

The beam-beam parameter would be the vector $\left(\xi_{1}, \xi_{2}\right)$.

\section{Existence and Uniqueness of a Solution to the Integral Equation}

We have to verify the three main hypotheses of the implicit function theorem, namely

1. $G: B \times I \rightarrow B$

2. $G$ is a continuously differentiable mapping of $B \times I$ into $B$.

3. $G_{f}\left(f_{0}, 0\right)^{-1}$ exists (i.e., $G_{f}\left(f_{0}, 0\right)$ is $1: 1$ and onto) and is bounded.

\subsection{Verifying Hypothesis 1}

Suppose that $f \in B$. Then $\mathbf{K} f(z)=\int K(z \mid y) f(y) d y$ exists and has continuous derivatives of all orders. For estimates of the action of $\mathbf{K}$ on $f$ we first note

\section{Lemma 1:}

$$
I=\int_{\mathbb{R}^{2}}\left|\frac{\partial^{m+n} K(z \mid y)}{\partial z_{1}^{m} \partial z_{2}^{n}}\right| \frac{d y}{1+|y|^{2 a}} \leq \frac{M_{m n}}{1+|z|^{2 a}}, \quad a>0,
$$

for any $m \geq 0, n \geq 0$, where the constant $M_{m n}$ depends on $a$ and the parameters defining $K$. Here and henceforth the Euclidean norm in any $\mathbb{R}^{n}$ is denoted by single bars: $|y|=\left(y^{T} y\right)^{1 / 2}$.

We prove Lemma 1 after three steps as follows. We use $M>0$ as a generic finite constant in majorizations; it may change value from one appearance to the next.

\section{Lemma 1a:}

$$
I=\int_{\mathbb{R}^{n}} \frac{\exp \left[-|z-y|^{2} / 2\right] d y}{1+|y|^{2 a}} \leq \frac{M}{1+|z|^{2 a}}, \quad a>0 .
$$

Proof 1a: With $0<\alpha<1$ we break the integral into $I_{+}$with $|y|>\alpha|z|$ and $I_{-}$with $|y|<\alpha|z|$. Then

$$
I_{+} \leq \frac{1}{1+(\alpha|z|)^{2 a}} \int_{|y|>\alpha|z|} \exp \left[-|z-y|^{2} / 2\right] d y \leq \frac{M}{1+|z|^{2 a}},
$$


whereas in $I_{-}$we have $|z-y| \geq|| z|-| y|| \geq(1-\alpha)|z|$, hence

$$
I_{-} \leq \exp \left[-(1-\alpha)^{2}|z|^{2} / 4\right] \int_{|y|<\alpha|z|} \exp \left[-|z-y|^{2} / 4\right] d y \leq \frac{M}{1+|z|^{2 a}}
$$

\section{Lemma 1b:}

If $A$ and $B$ are positive definite $n \times n$ matrices, then there is an $M$ such that for $a>0$ and all $z$ we have

$$
\frac{1}{1+\left(z^{T} A z\right)^{a}} \leq \frac{M}{1+\left(z^{T} B z\right)^{a}} .
$$

Proof 1b: We know that

$$
a_{-} \leq \frac{z^{T} A z}{z^{T} z} \leq a_{+}
$$

where $a_{\mp}$ is the minimum (maximum) eigenvalue of $A$, and similarly for $B$. Then $z^{T} A z \geq$ $\left(a_{-} / b_{+}\right) z^{T} B z$, and

$$
\frac{1}{1+\left(z^{T} A z\right)^{a}} \leq \frac{\beta}{\beta+\left(z^{T} B z\right)^{a}}, \quad \beta=\left(\frac{b_{+}}{a_{-}}\right)^{a} .
$$

We get a lower bound on the denominator of the r.h.s. if we replace its first term by 1 if $\beta \geq 1$, or multiply its second term by $\beta$ if $\beta<1$; thus $M=\max (1, \beta)$.

\section{Lemma 1c:}

$$
I=\int_{\mathbb{R}^{2}} \frac{K(z \mid y) d y}{1+|y|^{2 a}} \leq \frac{M}{1+|z|^{2 a}}, \quad a>0 .
$$

Proof 1c: Recall the definition of $K$ in (16) and (8) and put $\theta=2 \pi \nu$. Change the integration variable to $\zeta=\exp (A \theta) z^{\prime}$, noting (11). Then

$$
I=\frac{e^{2 \alpha \theta}}{2 \pi[\operatorname{det} \Sigma(\theta)]^{1 / 2}} \int \frac{\exp \left[-(z-\zeta)^{T} \Sigma(\theta)^{-1}(z-\zeta) / 2\right] d \zeta}{1+\left[\zeta^{T} e^{-A^{T} \theta} e^{-A \theta} \zeta\right]^{a}} .
$$

Since $\Sigma(\theta)^{-1}$ and $e^{-A^{T} \theta} e^{-A \theta}$ are positive definite, we can apply Lemma $1 \mathrm{~b}$ to get

$$
I \leq M \int \frac{\exp \left[-|z-\zeta|_{s}^{2} / 2\right] d \zeta}{1+|\zeta|_{s}^{2 a}}
$$

where we have introduced a new norm $|z|_{s}=\left(z^{T} \Sigma(\theta)^{-1} z\right)^{1 / 2}$. Lemma 1a holds as well if the Euclidean norm is replaced by the new norm. Applying Lemma 1b again, we then finish the proof.

Proof 1: We can now prove Lemma 1 by a similar argument. For $I$ of Lemma 1 we change the integration variable to $\zeta$ and apply Lemma $1 \mathrm{~b}$ as before to get

$$
I \leq M \int \frac{\left|P_{m n}(z-\zeta)\right| \exp \left[-|z-\zeta|_{s}^{2} / 2\right] d \zeta}{1+|\zeta|_{s}^{2 a}}
$$


where $P_{m n}(z)$ is a polynomial in the components of $z$. Now the r.h.s. can be estimated by the method used in the proof of Lemma 1a (but with the new norm), since the polynomial does not spoil convergence of the integrals bounding $I_{+}$and $I_{-}$. One more application of Lemma 1b finishes the proof.

Note also

Lemma 2: If $\phi: \mathbb{R}^{2} \rightarrow \mathbb{R}$ is any bounded function, $|\phi(z)| \leq b$, then

$$
\frac{1}{1+\left[q^{2}+(p+\phi(z))^{2}\right]^{a}} \leq \frac{M}{1+|z|^{2 a}} .
$$

Proof 2: Let $r(z)=\left(1+|z|^{2 a}\right) /\left(1+\left[q^{2}+(p+\phi(z))^{2}\right]^{a}\right)$. For $|p| \geq 2 b$ we have

$$
r(z) \leq \frac{1+\left(q^{2}+p^{2}\right)^{a}}{1+\left(q^{2}+p^{2} / 4\right)^{a}}<M
$$

whereas for $|p|<2 b$,

$$
r(z) \leq \frac{1+\left(q^{2}+(2 b)^{2}\right)^{a}}{1+q^{2 a}}<M
$$

To establish Hypothesis 1 , suppose that $f \in B$. Then

$$
|f(z)| \leq \frac{\|f\|}{w(z)}
$$

and $G(f, \xi)(z)$ is a continuous function of $z$, according to the definitions of $\mathbf{K}$ and $\mathbf{L}$. Also, $\mathbf{L} f$ of $(22)$ is bounded. Since $\int K\left(z^{\prime} \mid z^{\prime \prime}\right) d z^{\prime}=1$, we have

$$
|\mathbf{L} f(q)| \leq(2 \pi)^{3 / 2}\|f\| \iint \frac{K\left(z^{\prime} \mid z^{\prime \prime}\right) d z^{\prime} d z^{\prime \prime}}{w\left(z^{\prime \prime}\right)}<M\|f\| .
$$

Now by Lemma 1c, and Lemma 2 applied with $\phi=\xi \mathbf{L} f$, we see that $G(f, \xi)$ has finite norm and therefore belongs to $B$ :

$$
\begin{aligned}
\|G(f, \xi)\| & =\sup _{z} w(z)\left|f(z) \int f(u) d u-\mathbf{K} f(q, p+\xi \mathbf{L} f(q))\right| \\
& \leq M\|f\|^{2}+M\|f\| \sup _{z} \frac{w(z)}{w(q, p+\xi \mathbf{L} f(q))}<M .
\end{aligned}
$$

\section{$3.2 \quad$ Verifying Hypothesis 2}

Recall from (20) that $G$ has the form

$$
\begin{aligned}
& G(f, \xi)=G_{0}(f)-G_{1}(f, \xi) \\
& G_{0}(f)(z)=f(z) \int f(u) d u \\
& G_{1}(f, \xi)(z)=\mathbf{K} f(q, p+\xi \mathbf{L} f(q)),
\end{aligned}
$$


where the linear operator $\mathbf{L}$ is defined in (22). To verify Hypothesis 2 , we determine $G_{f}(f, \xi)$ and $G_{\xi}(f, \xi)$ as bounded linear operators and show that they are continuous functions of $(f, \xi)$ in the operator norm. The obvious candidates for these operators are obtained from the formal first-order variations of $G$ with respect to $f$ and $\xi$, respectively. Writing $h=\delta f$ for the variation of $f$, we find a linear integral operator $L=L_{0}+L_{1}+L_{2}: B \rightarrow B$ as the candidate for $G_{f}$ :

$$
\begin{aligned}
& \delta G_{0}=L_{0} \delta f, \quad \delta G_{1}=\left(L_{1}+L_{2}\right) \delta f \\
& {\left[L_{0}(f) h\right](z)=\int f(u) d u h(z)+f(z) \int h(u) d u} \\
& {\left[L_{1}(f, \xi) h\right](z)=\mathbf{K} h(q, p+\xi \mathbf{L} f(q))} \\
& {\left[L_{2}(f, \xi) h\right](z)=D_{2} \mathbf{K} f(q, p+\xi \mathbf{L} f(q)) \xi \mathbf{L} h(q),}
\end{aligned}
$$

where $D_{2}$ means derivative with respect to the second argument of a function $g(q, p)$. Similarly, the candidate $L_{3}: \mathbf{R} \rightarrow B$ for $G_{\xi}$ is multiplication by a function, that is

$$
\left[L_{3}(f, \xi) \kappa\right](z)=-\left[D_{2} \mathbf{K} f(q, p+\xi \mathbf{L} f(q)) \mathbf{L} f(q)\right] \kappa, \quad \kappa \in \mathbf{R} .
$$

The path to showing that $L=G_{f}$ is indicated right away in the case of the simple term $G_{0}$. We take $h, f \in B$ and compute

$$
\begin{aligned}
& \left\|G_{0}(f+h)-G_{0}(f)-L_{0}(f) h\right\|= \\
& \sup _{z} w(z)\left|h(z) \int h(u) d u\right| \leq\|h\|^{2} \int \frac{d u}{w(u)}=\mathcal{O}\left(\|h\|^{2}\right) .
\end{aligned}
$$

Also, $L_{0}(f)$ is bounded, since $\left\|L_{0}(f) h\right\| \leq M\|h\|$. Then $\left(G_{0}\right)_{f}(f)=L_{0}(f)$ by the definition (26). It is easy to see that $\left\|L_{0}(f)\right\|=\sup _{\|h\|=1}\left\|L_{0}(f) h\right\| \leq M\|f\|$. Then $L_{0}(f)$ is continuous, since it depends linearly on $f$.

Proceeding to the less trivial terms we define

$$
\zeta=z+(0, \xi \mathbf{L} f(q)), \quad \eta=(0, \xi \mathbf{L} h(q))=\mathcal{O}(\|h\|) .
$$

Suppressing reference to $\xi$ dependence, we have

$$
\begin{aligned}
& \left\|G_{1}(f+h)-G_{1}(f)-\left(L_{1}+L_{2}\right)(f) h\right\|= \\
& \left\|\mathbf{K}(f+h)(\zeta+\eta)-\mathbf{K} f(\zeta)-\mathbf{K} h(\zeta)-D_{2} K f(\zeta) \eta_{2}\right\| \leq T_{1}+T_{2},
\end{aligned}
$$

where

$$
\begin{aligned}
& T_{1}=\sup _{z} w(z)|\mathbf{K} h(\zeta+\eta)-\mathbf{K} h(\zeta)| \\
& \left.T_{2}=\sup _{z} w(z) \mid \mathbf{K} f(\zeta+\eta)\right)-\mathbf{K} f(\zeta)-\eta_{2} D_{2} \mathbf{K} f(\zeta) \mid .
\end{aligned}
$$

Now let us verify that both $T_{1}$ and $T_{2}$ are $\mathcal{O}\left(\|h\|^{2}\right)$. Considering first $T_{1}$, we apply the Taylor formula with remainder

$$
\phi(x+y)=\phi(x)+y \int_{0}^{1} \phi^{\prime}(x+u y) d u
$$


which gives

$$
\mathbf{K} h(\zeta+\eta)-\mathbf{K} h(\zeta)=\eta_{2} \int_{0}^{1} D_{2} \mathbf{K} h(\zeta+u \eta) d u
$$

Here $D_{2} \mathbf{K}$ can be estimated by Lemmas 1 and 2 as follows:

$$
\left|D_{2} \mathbf{K} h(\zeta+u \eta)\right| \leq \frac{M\|h\|}{w\left(q, \zeta_{2}+u \eta_{2}\right)} \leq \frac{M\|h\|}{w(z)},
$$

from which it follows that $T_{1}=\mathcal{O}\left(\|h\|^{2}\right)$. The argument to show that $T_{2}=\mathcal{O}\left(\|h\|^{2}\right.$ is similar, except that we must apply the second order Taylor formula,

$$
\phi(x+y)=\phi(x)+\phi^{\prime}(x) y+y^{2} \int_{0}^{1}(1-u) \phi^{\prime \prime}(x+u y) d u .
$$

Boundedness of $L_{1}(f)$ and $L_{2}(f)$ follows immediately from Lemmas 1 and 2 , and the fact that $\mathbf{L}$ is bounded as noted in (46). Thus $G_{f}=L$ as claimed.

The continuity of $G_{f}(f, \xi)$ is proved in a similar manner by Lemmas 1 and 2 and the first order Taylor formula. For instance, to prove continuity of $L_{2}$ we examine

$$
\begin{aligned}
& L_{2}\left(f+f_{1}, \xi+\xi_{1}\right)-L_{2}(f, \xi)= \\
& D_{2} \mathbf{K}\left(f+f_{1}\right)(\zeta+\phi)\left(\xi+\xi_{1}\right) \mathbf{L} h-D_{2} \mathbf{K} f(\zeta) \xi \mathbf{L} h= \\
& D_{2} \mathbf{K}\left(f+f_{1}\right)(\zeta+\phi) \xi_{1} \mathbf{L} h+D_{2} \mathbf{K} f_{1}(\zeta+\phi) \xi \mathbf{L} h+\int_{0}^{1} D_{2}^{2} \mathbf{K} f(\zeta+u \phi) d u \phi_{2},
\end{aligned}
$$

where

$$
\phi=\left(0, \xi_{1} \mathbf{L} f+\xi \mathbf{L} f_{1}\right) .
$$

The norm of each of the three terms in the last line of (59) is $\mathcal{O}\left(\|h\| \max \left(\left\|f_{1}\right\|,\left|\xi_{1}\right|\right)\right)$, which implies continuity, in fact Lipschitz continuity.

The same sort of argument shows that $G_{\xi}(f, \xi)=L_{3}(f, \xi)$ exists and is continuous.

\section{3 $\quad$ Verifying Hypothesis 3}

The hardest part of the proof is verifying item (3). In textbook examples it is usual to suppose that $G_{f}-1$ is a compact operator, in which case one can apply Fredholm theory to discuss existence of $G_{f}^{-1}$. In the present case this operator appears to be non-compact, and we have to resort to a more subtle method. We get the inverse by proving uniform convergence of an operator power series development. The convergence is at a slow rate determined by the small damping constant.

To check Hypothesis 3 we have to show that the equation

$$
G_{f}\left(f_{0}, 0\right) x=y
$$

has a unique solution $x \in B$ for any $y \in B$, and that the solution is a continuous function of $y$ in the norm topology of $B$. Since $f_{0}$ has unit integral, (61) has the form

$$
x(z)+f_{0}(z) \int x(u) d u-\mathbf{K} x(z)=y(z) .
$$


For the general problem (19), $G_{f}$ breaks into two independent blocks at zero current, and each block has the form (62). This is the reason that there is no substantial complication in extending our argument to the case of dissimilar beams.

To understand the structure of (62) it is important to note that for any $x \in B$,

$$
\int \mathbf{K} x(z) d z=\int x(z) d z
$$

Remark: In view of (63) we can see how essential it was to include $\int f(z) d z$ in the definition of $G$. If this factor were replaced by 1 then the second term on the l.h.s. of (62) would not be present, and there could be a solution of (61) only if $\int y(z) d z=0$, not for every $y \in B$.

It follows from (63) that any solution of (62) must satisfy

$$
\int x(z) d z=\int y(z) d z
$$

Consequently, any solution of (62) must also be a solution of

$$
\begin{aligned}
& x(z)=\mathbf{K} x(z)+p(z), \\
& p(z)=y(z)-f_{0}(z) \int y\left(z^{\prime}\right) d z^{\prime}, \quad \int p(z) d z=0 .
\end{aligned}
$$

We look for solutions of (62) among the solutions of (65). Iterating (65) $n-1$ times we find

$$
x=\mathbf{K}^{n} x+\sum_{m=1}^{n-1} \mathbf{K}^{m} p+p
$$

Here the story is different from the familiar case of the Neumann series, since the term $\mathbf{K}^{n} x$ does not vanish in the limit of large $n$. By the semigroup property of the linear Fokker-Planck evolution, $\mathbf{K}^{n}$ is given through (8) as follows:

$$
\mathbf{K}^{n} x(z)=\frac{1}{2 \pi \operatorname{det}^{1 / 2} \Sigma_{n}} \int \exp \left[-\frac{1}{2}\left|\Sigma_{n}^{-1 / 2}\left(z-e^{n A \theta} z^{\prime}\right)\right|^{2}\right] x\left(z^{\prime}\right) d z^{\prime},
$$

where

$$
\theta=2 \pi \nu, \quad \Sigma_{n}=\Sigma(n \theta) .
$$

If $x \in B$ the integral (68) converges uniformly in $n$, since the integrand is majorized by $\left|x\left(z^{\prime}\right)\right|$ and $\int|x(z)| d z<\infty$. We may then take the limit under the integral to obtain

$$
\lim _{n \rightarrow \infty} \mathbf{K}^{n} x(z)=f_{0}(z) \int x(z) d z
$$

since

$$
\Sigma_{n}=I-e^{n A \theta} e^{n A^{T} \theta} \rightarrow I
$$


follows from (9). Thus, from (67) and (70) we can hope to find a solution of (65) in the form of a series,

$$
x=f_{0} \int x\left(z^{\prime}\right) d z^{\prime}+\sum_{m=1}^{\infty} \mathbf{K}^{m} p+y-f_{0} \int y\left(z^{\prime}\right) d z^{\prime} .
$$

Any solution of (62) must satisfy (64), so that from (72) a candidate for a series solution is

$$
x(z)=\sum_{m=1}^{\infty} \mathbf{K}^{m} p(z)+y(z) .
$$

To establish that (73) is a unique solution to the original equation our main task will be to prove

Lemma 3: If $p \in B$ and $\int p(z) d z=0$ then

$$
\left|\mathbf{K}^{n} p(z)\right| \leq \frac{M e^{-n \theta \alpha}\|p\|}{w(z)} .
$$

Suppose that Lemma 3 is true, and that $y \in B$, hence $p \in B$. Then the following observations complete our argument:

(i) By (74) and the Weierstrass M-test, the series (73) converges uniformly in $z$ and therefore represents a continuous function, since its terms are continuous. The sum of the series is less in magnitude than $M \sum_{n} e^{-n \theta \alpha} / w(z)$, so it belongs to $B$.

(ii) Furthermore, (73) satisfies the original equation (62):

$$
\begin{aligned}
& \sum_{m=1}^{\infty} \mathbf{K}^{m} p+y+f_{0} \int\left[\sum_{m=1}^{\infty} \mathbf{K}^{m} p(u)+y(u)\right] d u-\sum_{m=2}^{\infty} \mathbf{K}^{m} p-\mathbf{K} y \\
& =\mathbf{K} p+y+f_{0} \int y(u) d u-\mathbf{K} y=y,
\end{aligned}
$$

since $\mathbf{K} f_{0}=f_{0}$ and $\int \sum_{m=1}^{\infty} \mathbf{K}^{m} p(u) d u=\sum_{m=1}^{\infty} \int \mathbf{K}^{m} p(u) d u=\sum_{m=1}^{\infty} \int p(u) d u=0$, the reversal of sum and integral in the latter being justified by (74).

(iii) To prove that the solution is unique, suppose that there were two solutions $x_{1}, x_{2}$ in $B$. Then $x=x_{1}-x_{2}$ satisfies (62) with $y=0$, from which it follows that $\int x(z) d z=0$, hence $x-\mathbf{K} x=0$. Iterating the latter equation, we have $x=\mathbf{K}^{n} x=\lim _{n \rightarrow \infty} \mathbf{K}^{n} x=$ $f_{0} \int x(z) d z=0$.

(iv) By (66), (73) and (74) we have $\|x\| \leq M\|p\|+\|y\| \leq M\|y\|$. Thus $G_{f}^{-1}\left(f_{0}, 0\right)$ is a bounded linear map, and $x$ is a continuous function of $y$ in the norm topology.

Proof 3: To motivate the first step in the proof of Lemma 3, note that if we could take the limit $n \rightarrow \infty$ under the integral that defines $\mathbf{K}^{n} p$, the limit of that integral would be $f_{0}(z) \int p\left(z^{\prime}\right) d z^{\prime}$. This indicates that the above noted property $\int p(z) d z=0$ is essential to 
convergence of the series in (73). Accordingly we formally subtract a term that is zero to obtain

$$
\begin{aligned}
\left|\mathbf{K}^{n} p(z)\right| & =\frac{1}{2 \pi \operatorname{det}^{1 / 2} \Sigma_{n}}\left|\int\left[e^{-|x-y|^{2} / 2}-e^{-|x|^{2} / 2}\right] p\left(z^{\prime}\right) d z^{\prime}\right| \\
& \leq \frac{M\|p\| e^{-|x|^{2} / 2}}{\operatorname{det}^{1 / 2} \Sigma_{n}} \int\left|e^{-|y|^{2} / 2+x^{T} y}-1\right| \frac{d z^{\prime}}{w\left(z^{\prime}\right)}
\end{aligned}
$$

where

$$
y=e^{-n \alpha \theta} \Sigma_{n}^{-1 / 2} R(n \theta) z^{\prime}, \quad x=\Sigma_{n}^{-1 / 2} z .
$$

Notice that

$$
\left|z^{\prime}\right|^{2}=e^{2 n \alpha \theta} y^{T} S_{n} y>s e^{2 n \alpha \theta}|y|^{2}, \quad S_{n}=\Sigma_{n}^{1 / 2}\left(R(n \theta) R^{T}(n \theta)\right)^{-1} \Sigma_{n}^{1 / 2}
$$

where $s>0$ is an $n$-independent lower bound on the eigenvalues of $S_{n}$ as given in (118). Also,

$$
\sigma|x|^{2}<|z|^{2}<|x|^{2}
$$

where $\sigma>0$ and 1 are n-independent lower and upper bounds, respectively, on the eigenvalues of $\Sigma_{n}$. A value for $\sigma$ is given in (116). In estimates we repeatedly use (79) to relate $\left|z^{\prime}\right|$ and $|y|$ and (80) to relate $|z|$ and $|x|$. Now note that $e^{v}-1=v \int_{0}^{1} e^{v t} d t$ and that $-|y|^{2} / 2+x^{T} y=-|y-x|^{2} / 2+|x|^{2} / 2 \leq|x|^{2} / 2$, so that by (79) and (80) we have

$$
\left|e^{-|y|^{2} / 2+x^{T} y}-1\right| \leq\left.|-| y\right|^{2} / 2+x^{T} y \mid \int_{0}^{1} e^{t|x|^{2} / 2} d t \leq M e^{-n \alpha \theta}\left(\left|z^{\prime}\right|^{2}+|z|\left|z^{\prime}\right|\right) \frac{e^{|x|^{2} / 2}-1}{|x|^{2}}
$$

Using this in (77) and noting that $\operatorname{det} \Sigma_{n}>\sigma^{2}$ we find

$$
\begin{aligned}
\left|\mathbf{K}^{n} p(z)\right| & \leq M e^{-n \alpha \theta}\|p\|(1+|z|) \frac{1-e^{-|x|^{2} / 2}}{|x|^{2}} \\
& \leq M e^{-n \alpha \theta}\|p\|(1+|z|) \frac{1-e^{-|z|^{2} /(2 \sigma)}}{|z|^{2}}
\end{aligned}
$$

The condition $a>2$ ensured convergence of the $z^{\prime}$-integral. (The requirement $a>2$ in the definition of the norm in fact arose from this estimate). Now the bound (82) is enough to show that the series of (73) converges uniformly in the maximum norm for $z$ in any compact region and therefore defines a continuous function of $z$. Since it gives decay only as $1 /|z|$ it is insufficient to show that the sum belongs to $B$.

To complete the job we get a bound by a different method which fails at small $|z|$ but works for $|z|>r$. For that we begin with (77) and break the integral $I$ into a part $I_{-}$with $|y|<b|x|$ and a part $I_{+}$with $|y|>b|x|$, where $0<b<1 / 2$. For $I_{-}$we apply the bounds

$$
x^{T} y-|y|^{2} / 2 \leq b|x|^{2}, \quad\left|e^{x^{T} y-|y|^{2} / 2}-1\right| \leq\left.\left|x^{T} y-\right| y\right|^{2} / 2 \mid \int_{0}^{1} e^{t b|x|^{2}} d t
$$


which leads as in the calculation above to

$$
\left|I_{-}\right| \leq M e^{-n \alpha \theta}\|p\| e^{-(1 / 2-b)|z|^{2}}|z| \leq M \frac{e^{-n \alpha \theta}\|p\|}{w(z)} .
$$

In $I_{+}$we do not get the decay at large $n$ from the coefficient of $p\left(z^{\prime}\right)$, but rather from the decay of $p\left(z^{\prime}\right)$ at large $z^{\prime}$. Accordingly we replace the minus sign in (77) by plus, and treat the two terms separately: $\left|I_{+}\right| \leq M\|p\|\left(J_{1}+J_{2}\right)$ where

$$
J_{1}=e^{-|x|^{2} / 2} \int_{|y|>b|x|} \frac{d z^{\prime}}{w\left(z^{\prime}\right)}, \quad J_{2}=\int_{|y|>b|x|} \frac{e^{-|x-y|^{2} / 2} d z^{\prime}}{w\left(z^{\prime}\right)} .
$$

We change the variable of integration in $J_{1}$ to $y$ and apply (79) and (80). The determinant of the Jacobian is less than or equal to $\exp (2 n \alpha \theta)$ since $\operatorname{det} R=1$. We find

$$
\begin{aligned}
J_{1} & \leq \quad e^{-|x|^{2} / 2} e^{2 n \alpha \theta} \int_{|y|>b|x|} \frac{d y}{1+e^{2 a n \alpha \theta}\left(y^{T} S_{n} y\right)^{a}} \\
& \leq \quad M e^{-|z|^{2} / 2} e^{-2(a-1) n \alpha \theta} \int_{|y|>b r} \frac{d y}{|y|^{2 a}} \leq M \frac{e^{-n \alpha \theta}}{w(z)}
\end{aligned}
$$

For $J_{2}$ we proceed in the same way to get

$$
\begin{aligned}
J_{2} & \leq M e^{-2(a-1) n \alpha \theta} \int_{|y|>b|x|} \frac{e^{-|x-y|^{2} / 2} d y}{|y|^{2 a}} \leq \frac{M e^{-2(a-1) n \alpha \theta}}{(b|z|)^{2 a}} \int_{\mathbb{R}^{2}} e^{-|x-y|^{2} / 2} d y \\
& \leq M \frac{e^{-n \alpha \theta}}{w(z)}, \quad|z|>r .
\end{aligned}
$$

Now by applying (82) for $|z| \leq r$ and $(34,86,87)$ for $|z|>r$, we complete the proof of Lemma 3.

\section{The Question of Positivity}

We derived the integral equation (21) as a necessary condition on the equilibrium state of colliding electron beams. The equation has a unique positive solution at zero beam current, the Gaussian of Eq.(23). We proved that this solution has a unique continuation $f(z, \xi)$ to non-zero current, with $\int f(z, \xi) d z=1$. We have not yet proved that $f(z, \xi) \geq 0$, as is required of a probability density. Positivity would follow if we knew that the following iteration converged to $f=\mathcal{A}(f) \in B$ at sufficiently small current:

$$
f^{(n+1)}=\mathcal{A}\left(f^{(n)}, \xi\right), \quad f^{(0)}=f_{0}
$$

where the definition of $\mathcal{A}$ is obtained from (21),

$$
\mathcal{A}(f, \xi)(z)=\int K\left(q, p+\xi \mathbf{L} f(q) \mid z^{\prime}\right) f\left(z^{\prime}\right) d z^{\prime}
$$




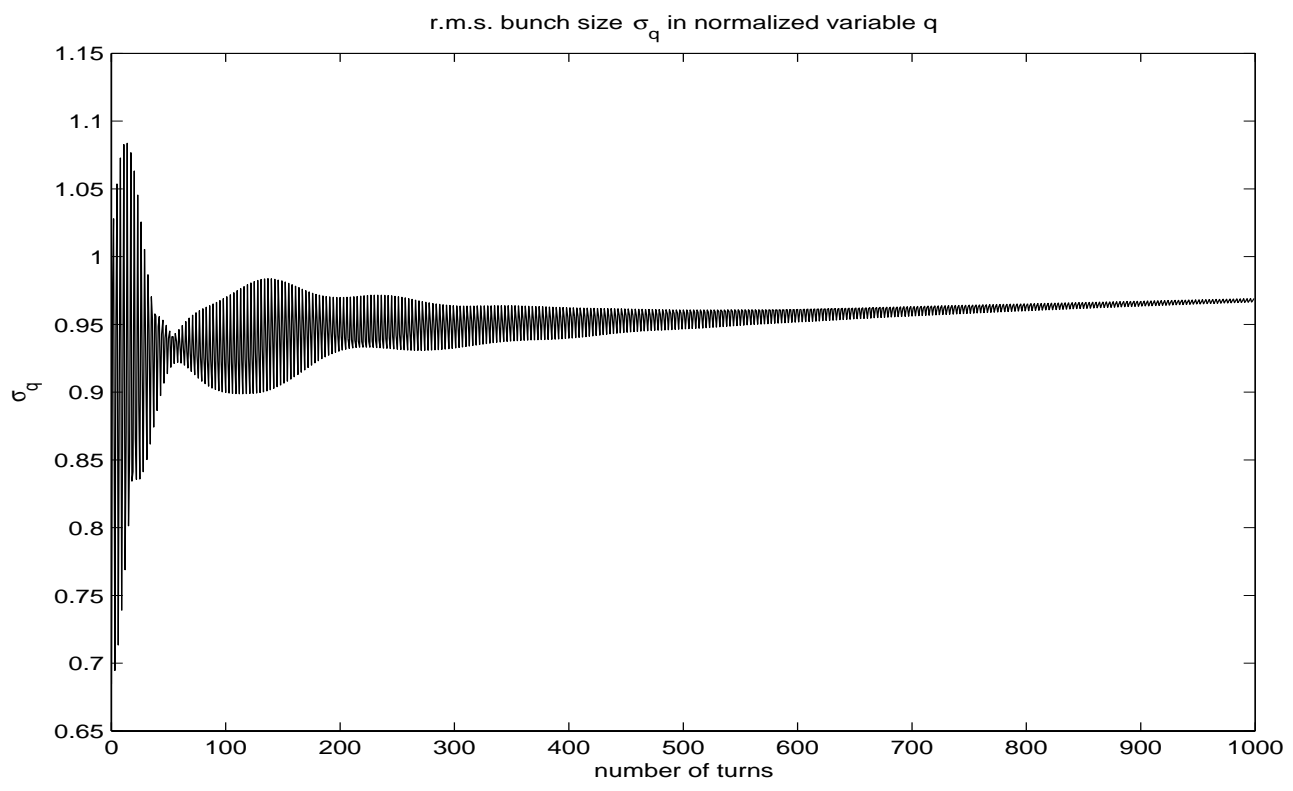

Figure 1: Bunch size $\sigma_{q}$, first 1000 turns

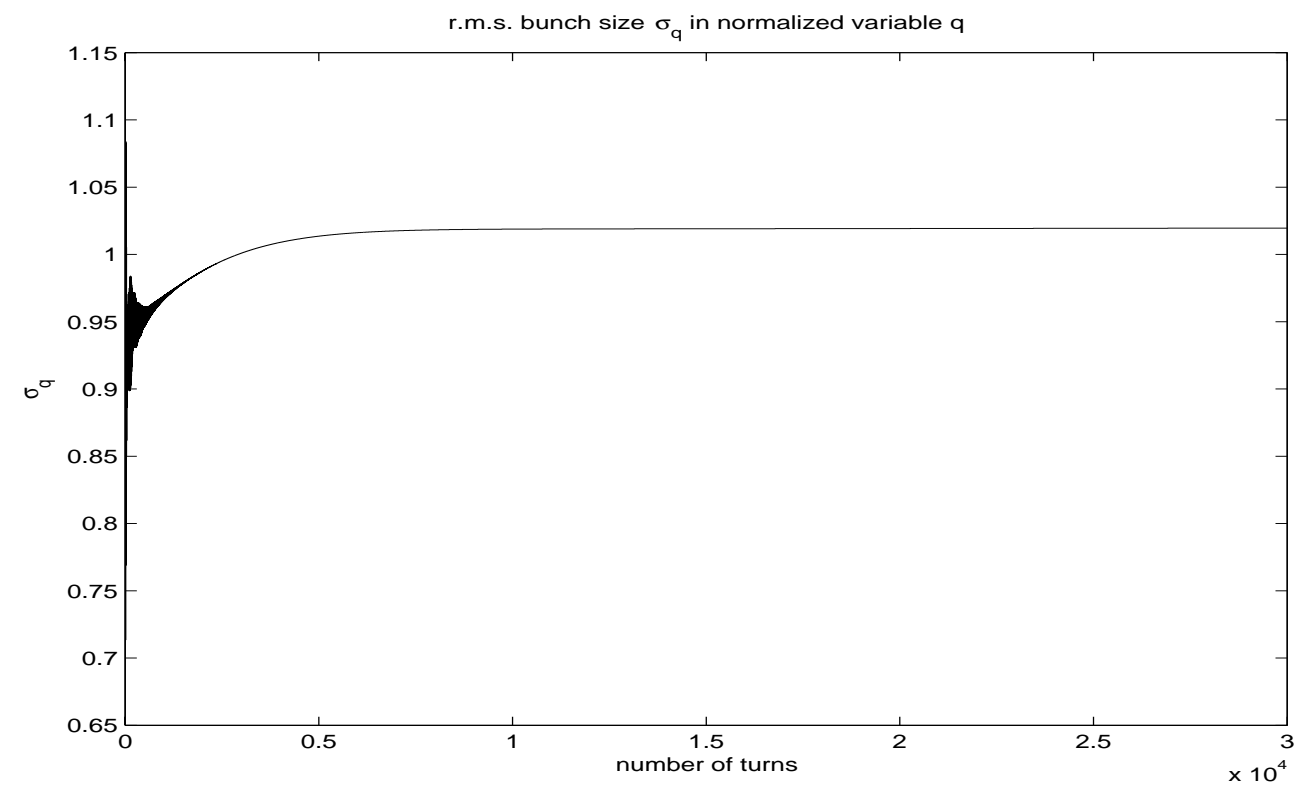

Figure 2: Bunch size $\sigma_{q}$, first 30000 turns 


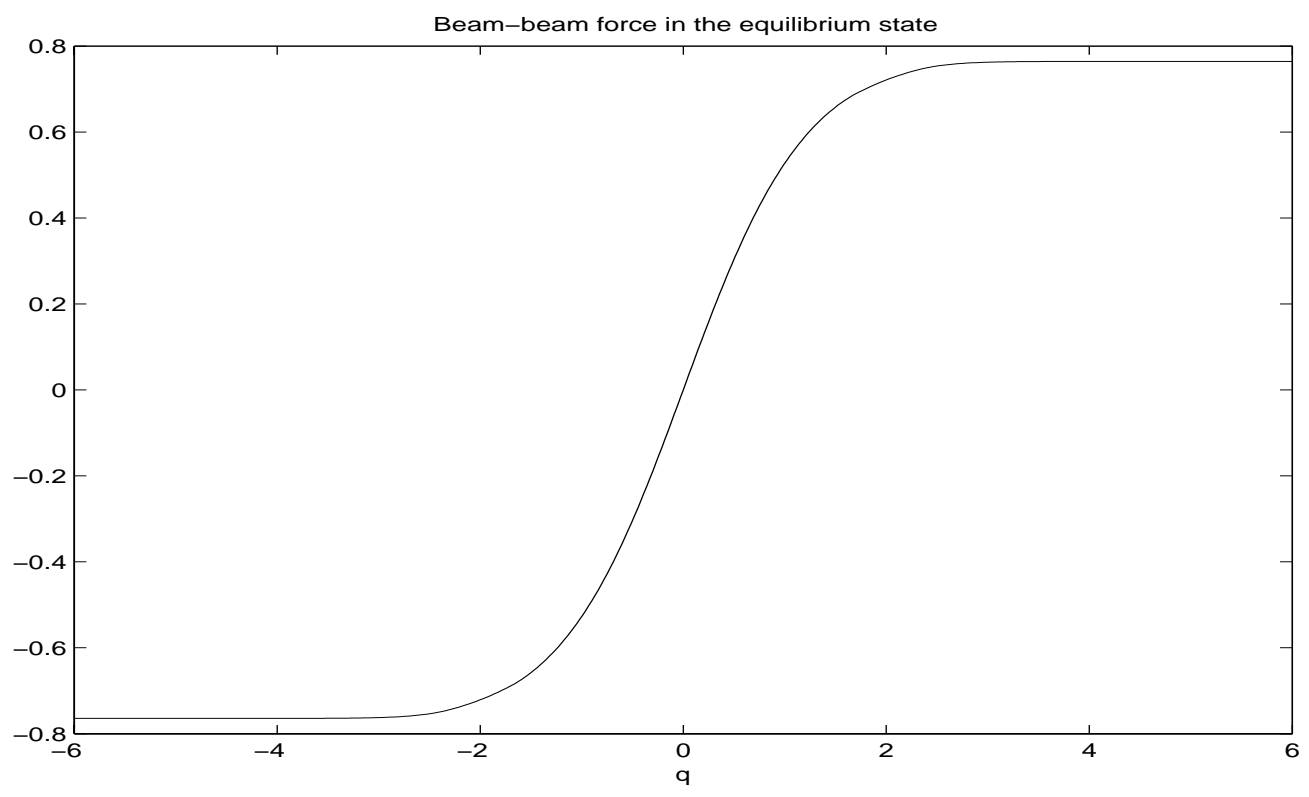

Figure 3: Beam-beam force in equilibrium state

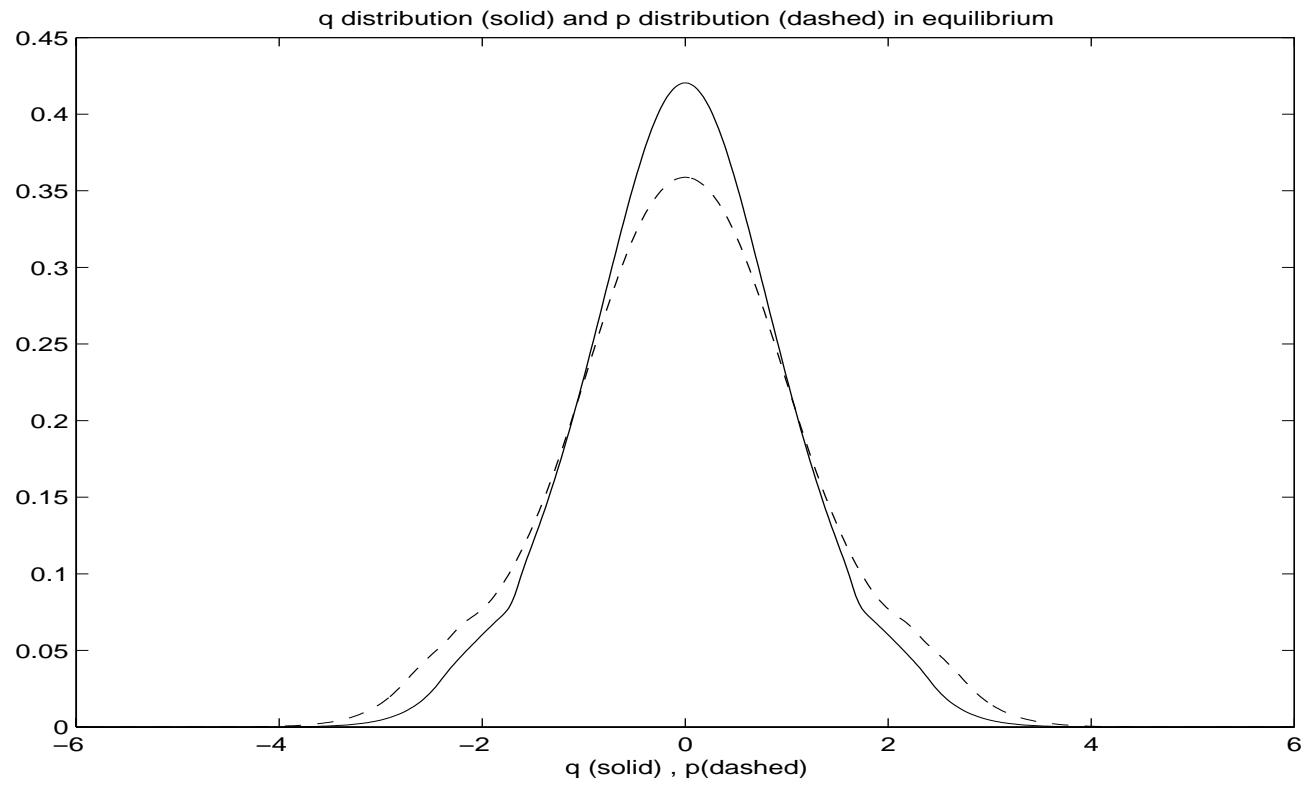

Figure 4: $\int f(q, p) d p$ (solid) and $\int f(q, p) d q$ (dashed) in the equilibrium state. 


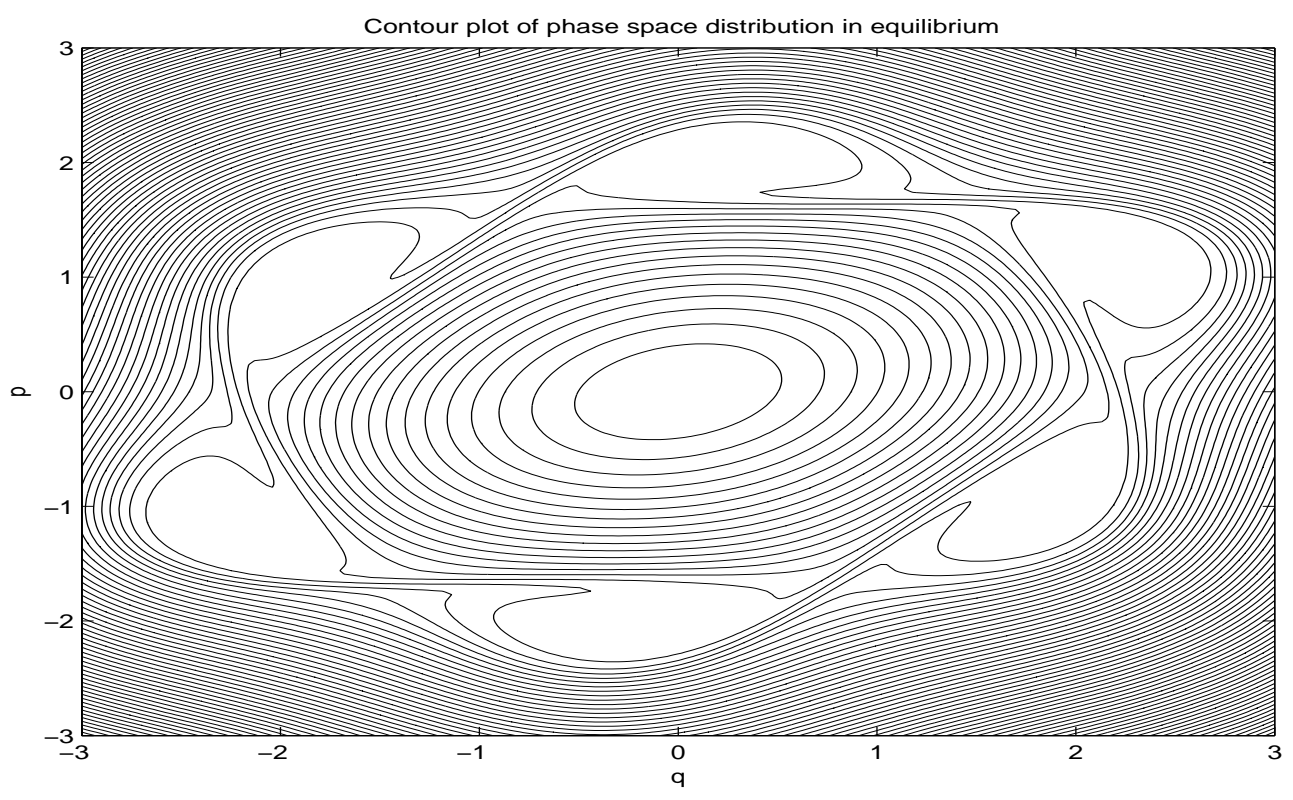

Figure 5: Contour plot of $f(q, p)$ in the equilibrium state.

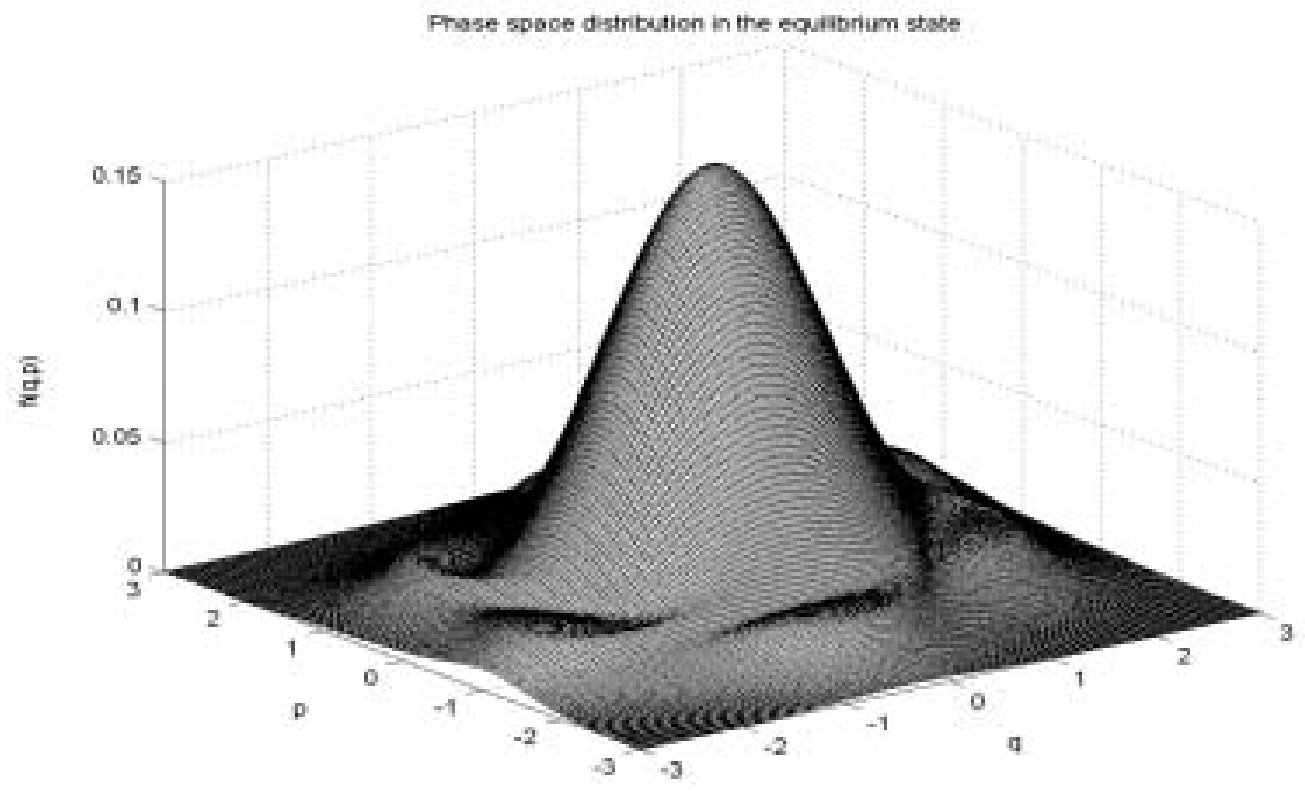

Figure 6: 3-D plot of $f(q, p)$ in the equilibrium state. 
The operator $\mathcal{A}$ preserves both positivity and the integral of a function to which it is applied. Consequently, convergence as stated, beginning with the positive zero-current solution $f_{0}$, would produce a positive solution, which must be identical to the solution established by the implicit function theorem.

At present we have no mathematical understanding of a mechanism for convergence of (88), although convergence would be perfectly in accord with physical intuition about equilibration. Furthermore, we have numerical evidence of convergence, as first reported in [8]. In the numerical work the Fokker-Planck evolution between beam-beam kicks was done by numerical solution of the partial differential equation (2). That is equivalent, modulo discretization error, to applying the propagator (16) as we have done in the present work. An effort was made to control discretization error, but whatever the deficiencies in that regard, the convergence of the iteration to an equilibrium was found to very robust. We give some results for a case of identical beams with $\nu=0.6364, \beta^{*}=0.015 \mathrm{~m}, \epsilon=1.44 \cdot 10^{-9} \mathrm{~m}, \xi=$ $0.0485, n_{d}=5000$. The numerical method is that of [8], with a $401 \times 401$ grid in phase space. Figure 1 shows the r.m.s. vertical bunch size $\sigma_{q}$ in the normalized variable $q$, versus turn number, for the first 1000 turns. The plot shows rapid oscillations with a period of about 3 turns, which begin to damp out by 1000 turns. Figure 2 shows the same quantity over 30000 turns. The number of turns for disappearance of oscillations and convergence to an equilibrium is 2 or 3 times the radiation damping time of the machine ( $n_{d}$ turns), again in agreement with intuition. The beam-beam force, proportional to $\mathbf{L} f(q)$, is shown in Figure 3 as it looks in the equilibrium state at 30000 turns. The corresponding distributions in $q$ and $p$ are plotted in Figure 4. Since $d / d q \int \operatorname{sgn}\left(q-q^{\prime}\right) \rho\left(q^{\prime}\right) d q^{\prime}=2 \rho(q)$, the beam-beam force is proportional to the integral of the $q$-distribution. The shoulders in the plots of Figure 4 seem to be associated with a broad sixth-order resonance, as is revealed by the contour plot of $f(q, p)$ given in Figure 5 , and the three-dimensional plot of the same in Figure 6 . The distribution is extremely flat in six regions, apparently vestiges of the $\nu=2 / 3$ Hamiltonian resonance islands as perturbed by damping and noise.

\section{Conclusion}

We have derived the integral equation (21) as a necessary condition on the equilibrium phase space distribution for the Chao-Ruth model of colliding electron beams. We proved that this equation has a unique solution in the function space defined through (29) when the beam current is sufficiently small. We are fairly confident that the proof will go through in almost the same way for other models in one degree of freedom [5] and for the model in two degrees of freedom in which the force is obtained from the two-dimensional Poisson equation. A sideline of our analysis was the probabilistic derivation of an elegant formula

for the fundamental solution of the Fokker-Planck equation with harmonic force. We expect this formula to have further applications. 


\section{A Fundamental Solution of the Fokker-Planck Equation with Harmonic Force}

Our definition of phase space coordinates in (1) is a bit unusual in that it involves the emittance $\epsilon$, which has a statistical definition. For the present discussion let us start with conventional coordinates having a purely mechanical definition, namely $\zeta=(Q, P)=\sqrt{\epsilon}(q, p)$. For the betatron motion unperturbed by the beam-beam force and radiation, the CourantSnyder action $J$ is invariant, where

$$
J=\frac{1}{2 \beta(s)}\left[x^{2}+\left(\beta(s) p-\beta^{\prime}(s) x / 2\right)^{2}\right]=\frac{1}{2}\left[Q^{2}+P^{2}\right] .
$$

With time coordinate $\theta=\int_{0}^{s} d u / \beta(u)$ this quantity is also the Hamiltonian $H$ for $\zeta$. The corresponding Fokker-Planck equation for the probability density $\psi(\zeta, \theta)$ may be written as

$$
\frac{\partial \psi}{\partial \theta}+\nabla \cdot(A \zeta \psi)=D \frac{\partial^{2} \psi}{\partial P^{2}}
$$

where $\nabla \cdot$ is the divergence in $\mathbb{R}^{2}$, and the matrix $A$ is

$$
\left(\begin{array}{rr}
0 & 1 \\
-1 & -2 \alpha
\end{array}\right)
$$

The damping and diffusion constants are $\alpha$ and $D$, respectively. The differential equation for damped harmonic motion is $d \zeta / d \theta=A \zeta$, and $e^{A \theta}$ as given in (9) is the corresponding principal solution matrix (or transfer matrix in the nomenclature of accelerator physics). Rearranging (91) we have

$$
\frac{\partial \psi}{\partial \theta}+P \frac{\partial \psi}{\partial Q}-Q \frac{\partial \psi}{\partial P}=2 \alpha \frac{\partial}{\partial P}(P \psi)+D \frac{\partial^{2} \psi}{\partial P^{2}} .
$$

We look for the $\theta$-independent equilibrium solution $\psi_{0}$ as an even function of $P$, in accord with the principle of detailed balance $[16,17]$. Then the left hand side of $(93)$ is odd in $P$, while the right hand side is even, therefore either side is zero. The left side set to zero says $\left[\psi_{0}, H\right]=0$, where [ ] is the Poisson bracket, which is satisfied by any smooth function $\psi_{0}(Q, P)=\Phi(H)$. Putting this in the right side set to zero we have

$$
2 \alpha \Phi+D \Phi^{\prime}+P^{2}\left[2 \alpha \Phi^{\prime}+D \Phi^{\prime \prime}\right]=0 .
$$

Since this equation must hold for all $Q$ at $P=0$, a solution must have the form $\Phi(H)=$ const $\cdot \exp (-2 \alpha H / D)$, and indeed this is the general solution for all $(Q, P)$. Normalizing $\psi_{0}$ to have unit integral, we have

$$
\psi_{0}(Q, P)=\frac{1}{2 \pi \epsilon} \exp \left(-\frac{J}{\epsilon}\right), \quad \epsilon=\frac{D}{2 \alpha} .
$$

One easily verifies that $\epsilon=\langle J\rangle$, the expected value of $J$ in the unperturbed equilibrium state. Our definition of emittance as $\epsilon=\langle J\rangle$ agrees with Ref.[10], but one finds several 
different but related definitions in the literature. Rewriting (93) in terms of the thoroughly normalized dimensionless variables $(q, p)=(Q, P) / \sqrt{\epsilon}$, we find the equation for $f(q, p, \theta)=$ $\epsilon \psi(Q, P, \theta)$ previously stated in $(2)$ :

$$
\frac{\partial f}{\partial \theta}+p \frac{\partial f}{\partial q}-q \frac{\partial f}{\partial p}=2 \alpha \frac{\partial}{\partial p}\left[p f+\frac{\partial f}{\partial p}\right] .
$$

It appears in (96) that the constant $D$ has been eliminated, but this is true only at the level of solving the differential equation. To get results concerning the physical variables $\left(y, y^{\prime}\right)$ one needs as well the ratio $\epsilon=D / 2 \alpha$ in (1).

The Fokker-Planck equation (96) gives the evolution law of the probability density of the stochastic process $Z(\theta)$ defined by the Langevin equation

$$
\frac{d Z}{d \theta}=A Z+2 \sqrt{\alpha}\left(\begin{array}{l}
0 \\
1
\end{array}\right) \xi(\theta),
$$

where $\xi(\theta)$ is Gaussian white noise. Formally, $\xi(\theta)$ is the derivative of the Wiener process $W(\theta)$ (mathematical Brownian motion), but that derivative actually fails to exist almost everywhere. The associated Itô stochastic differential equation [15] is

$$
d Z=A Z d t+2 \sqrt{\alpha}\left(\begin{array}{c}
0 \\
1
\end{array}\right) d W(\theta) .
$$

The solution of (98) is

$$
Z(\theta)=e^{A \theta}\left[Z(0)+2 \sqrt{\alpha} \int_{0}^{\theta} e^{-A t}\left(\begin{array}{l}
0 \\
1
\end{array}\right) d W(t)\right],
$$

where the second term has a technical definition as the so-called Itô integral. It can be shown that the Itô integral is a Gaussian random variable. We assume that $Z(0)$ is either deterministic or is a Gaussian random variable independent of $W(\theta)$; then $Z(\theta)$ is a Gaussian variable. Furthermore, $f$ is the probability density of $Z$, so that

$$
f(z, \theta)=\frac{1}{2 \pi \operatorname{det}^{1 / 2} C(\theta)} \exp \left[-\frac{1}{2}(z-m(\theta))^{T} C(\theta)^{-1}(z-m(\theta))\right],
$$

where $m$ and $C$ are the vector mean and covariance matrix,

$$
\begin{aligned}
& m(\theta)=E[Z(\theta)] \\
& C(\theta)=E\left[(Z(\theta)-m(\theta))(Z(\theta)-m(\theta))^{T}\right]=M(\theta)-m(\theta) m(\theta)^{T}, \\
& M(\theta)=E\left[Z(\theta) Z(\theta)^{T}\right] .
\end{aligned}
$$

Since the expected value of the integral in (99) is zero, we have

$$
m(t)=e^{A \theta} E[Z(0)],
$$


and $M(\theta)$ satisfies the differential equation

$$
\frac{d M}{d \theta}=A M+M A^{T}+4 \alpha\left(\begin{array}{ll}
0 & 0 \\
0 & 1
\end{array}\right) .
$$

Before outlining the derivation of (103), we discuss its solution and the resulting $C(\theta)$. The general solution is

$$
M(\theta)=e^{A \theta} K e^{A^{T} \theta}+M_{p}(\theta),
$$

where $K$ is an arbitrary constant matrix and $M_{p}$ is a particular solution. Using (101) and (102), and determining $K$ in terms of the initial value of $C$, we have

$$
\begin{aligned}
& m(\theta)=e^{A \theta} m(0), \\
& C(\theta)=e^{A \theta}\left(C(0)-M_{p}(0)\right) e^{A^{T} \theta}+M_{p}(\theta) .
\end{aligned}
$$

These expressions substituted in (100) complete the solution of the Fokker-Planck equation (96) by the probabilistic method. One can check by direct substitution that (100) satisfies (96), if $\operatorname{det} C \neq 0$.

Equation (103) can be established by applying Itô's formula to the stochastic differential equation. Here we give an heuristic derivation, starting with the solution (99). We have

$$
\begin{aligned}
& M(\theta)=E\left[Z(\theta) Z^{T}(\theta)\right]=e^{A \theta} E\left[\left(Z(0)+2 \sqrt{\alpha} \int_{0}^{\theta} e^{-A t}\left(\begin{array}{c}
0 \\
1
\end{array}\right) d W(t)\right) .\right. \\
& \left.\left(Z(0)^{T}+2 \sqrt{\alpha} \int_{0}^{\theta}\left(\begin{array}{ll}
0 & 1
\end{array}\right) e^{-A^{T} s} d W(s)\right)\right] e^{A^{T} \theta} \\
& =e^{A \theta}\left[M(0)+4 \alpha \int_{0}^{\theta} d t \int_{0}^{\theta} d s e^{-A t}\left(\begin{array}{cc}
0 & 0 \\
0 & 1
\end{array}\right) e^{-A^{T} s} E[\xi(t) \xi(s)]\right] e^{A^{T} \theta} \\
& =e^{A \theta}\left[M(0)+4 \alpha \int_{0}^{\theta} e^{-A t}\left(\begin{array}{cc}
0 & 0 \\
0 & 1
\end{array}\right) e^{-A^{T} t} d t\right] e^{A^{T} \theta} .
\end{aligned}
$$

Here we have applied $E[\xi(t) \xi(s)]=\delta(t-s)$, which is a formal expression of a basic property of the stochastic integral. That is, the autocorrelation function of white noise is the delta function. Now (103) follows from differentiation of (107).

Now consider our special case with $A$ defined in (92), for which a particular solution of (103) is the unit matrix $M_{p}=I$. With that choice in (106) we see from (9) that $C(\theta) \rightarrow$ $I, m(\theta) \rightarrow 0, \theta \rightarrow \infty$, hence $f(z, \theta) \rightarrow f_{0}(z)$. That is, whatever the initial condition the solution tends to the equilibrium. The fundamental solution $\Phi\left(z, z_{0}, \theta\right)$ of the Fokker-Planck equation is (100) with a non-random initial condition $Z(0)=z_{0}$. In this case $m(0)=E\left[z_{0}\right]=$ $z_{0}$ and $C(0)=0$, so that $C(\theta)=I-e^{A \theta} e^{A^{T} \theta}$ coincides with $\Sigma(\theta)$, and $\Phi$ is indeed as claimed in (8). The right hand side of $(7)$ is a solution which tends to $f(z, 0)$ as $\theta \rightarrow 0$, since $\Phi$ peaks up to form a delta function $\delta\left(z-z^{\prime}\right)$ in the limit. 


\section{B Eigenvalues of $R R^{T}$}

We are concerned with the matrix

$$
\Sigma(\theta)=I-e^{-2 \alpha \theta} R(\theta) R^{T}(\theta)
$$

with $R$ defined in (10) and (11). One can prove by a probabilistic argument that $\Sigma(\theta)$ is positive-definite for $\theta>0$. Here we wish to prove the same thing by a direct examination of eigenvalues, and also derive some bounds needed in the proof of Lemma 3. We first calculate the eigenvalues of $R R^{T}$ from (10), beginning with the trace

$$
\operatorname{tr}\left[R R^{T}\right]=2\left(1+2\left(\frac{\alpha z}{\Omega}\right)^{2}\right), \quad z=\sin (\Omega \theta) .
$$

Since $\operatorname{det} R R^{T}=1$ this gives the eigenvalues of $R R^{T}$ as

$$
\begin{aligned}
& \lambda_{ \pm}(z)=1+2\left(\frac{\alpha z}{\Omega}\right)^{2} \pm\left[\left(1+2\left(\frac{\alpha z}{\Omega}\right)^{2}\right)^{2}-1\right]^{1 / 2} \\
& =\left(1+\left(\frac{\alpha z}{\Omega}\right)^{2}\right)+\left(\frac{\alpha z}{\Omega}\right)^{2} \pm 2 \frac{\alpha|z|}{\Omega}\left(1+\left(\frac{\alpha z}{\Omega}\right)^{2}\right)^{1 / 2} \\
& =\frac{1}{\Omega^{2}}\left[\alpha|z| \pm\left(\Omega^{2}+(\alpha z)^{2}\right)^{1 / 2}\right]^{2}
\end{aligned}
$$

where it is understood that all square roots are positive. The eigenvalues of $\Sigma$ are

$$
\sigma_{\mp}(\theta)=1-e^{-2 \alpha \theta} \lambda_{ \pm}(z) \text {. }
$$

We compute their derivatives as follows:

$$
\sigma_{\mp}^{\prime}(\theta)=e^{-2 \alpha \theta}\left[2 \alpha \lambda_{ \pm}(z)-\lambda_{ \pm}^{\prime}(z) \Omega \cos (\Omega \theta)\right], \quad \lambda_{ \pm}^{\prime}(z)= \pm \frac{2 \operatorname{sgn}(z) \alpha \lambda_{ \pm}(z)}{\left(\Omega^{2}+(\alpha z)^{2}\right)^{1 / 2}}
$$

This gives

$$
\sigma_{\mp}^{\prime}(\theta)=2 \alpha \lambda_{ \pm}(z) e^{-2 \alpha \theta}\left[1 \mp \operatorname{sgn}(z) \frac{\Omega \cos (\Omega \theta)}{\left[\Omega^{2}+(\alpha z)^{2}\right]^{1 / 2}}\right] \geq 0 .
$$

For $0<\theta<\pi / \Omega$ we see that $\sigma_{\mp}$ is definitely increasing, and for larger $\theta$ it is non-decreasing (actually increasing except for periodically recurring points of zero slope). It follows that $\Sigma(\theta)$ is positive-definite for positive $\theta$, since $\sigma_{\mp}(0)=0$.

For bounds on $\lambda_{ \pm}$note that (110) gives

$$
\lambda_{-} \leq 1 \leq \lambda_{+}
$$

From the sign of $\lambda_{ \pm}^{\prime}$ given in (112) we can find an upper bound on $\lambda_{+}$and a lower bound on $\lambda_{-}$. Altogether,

$$
\frac{1-\alpha}{1+\alpha} \leq \lambda_{-} \leq 1 \leq \lambda_{+} \leq \frac{1+\alpha}{1-\alpha}
$$


For the proof of Lemma 3 we are concerned with $\Sigma_{n}=\Sigma(n \theta), \theta=2 \pi \nu, n=1,2, \cdots$. Choosing a $\theta_{0} \in(0, \pi / \Omega)$ such that $\theta_{0}<2 \pi \nu$ we have by (113) that

$$
0<\sigma=\sigma_{-}\left(\theta_{0}\right)<\sigma_{\mp}(n \theta)<1 .
$$

We also need a lower bound on the eigenvalues of the matrix $S_{n}$ appearing in (79), namely

$$
S_{n}=\Sigma_{n}^{1 / 2}\left(R(n \theta) R(n \theta)^{T}\right)^{-1} \Sigma_{n}^{1 / 2}, \quad \theta=2 \pi \nu .
$$

Since $\Sigma_{n}$ and $R(n \theta) R(n \theta)^{T}$ are diagonalized by the same orthogonal transformation, the eigenvalues of $S_{n}$ are

$$
\frac{\sigma_{\mp}}{\lambda_{ \pm}}>\frac{\sigma}{\lambda_{+}} \geq \frac{1-\alpha}{1+\alpha} \sigma=s>0
$$

\section{Completeness of the space $B$}

We wish to show that the space $B$ defined in Section 2, with norm given in (29), is complete. Supposing that $\left\{f_{n}\right\}$ is a Cauchy sequence in $B$, we must show that there exists $f \in B$ such that $\left\|f_{n}-f\right\| \rightarrow 0$.

Step 1: Construction of a candidate for $f$.

For any fixed $z,\left\{f_{n}(z)\right\}$ is a Cauchy sequence in $\mathbb{R}$ since $\left|f_{n}(z)-f_{m}(z)\right| \leq w(z) \mid f_{n}(z)-$ $f_{m}(z) \mid \leq\left\|f_{n}-f_{m}\right\|$. Since $\mathbb{R}$ is complete there exists an $f$ such that $f_{n}(z) \rightarrow f(z)$, that is, $f_{n}$ converges pointwise to $f$.

Step 2: $\|f\|<\infty$.

$\left\{\left\|f_{n}\right\|\right\}$ is a Cauchy sequence in $\mathbf{R}$ since by the triangle inequality $\left|\left\|f_{n}\right\|-\left\|f_{m}\right\|\right| \leq\left\|f_{n}-f_{m}\right\|$. Thus $w(z)\left|f_{n}(z)\right| \leq\left\|f_{n}\right\|$ implies $w(z)|f(z)| \leq \lim _{n \rightarrow \infty}\left\|f_{n}\right\|=: d<\infty$. Therefore, $\|f\| \leq d$.

Step 3: $\left\|f_{n}-f\right\| \rightarrow 0$.

Since $w(z)\left|f_{n}(z)-f(z)\right| \leq w(z)\left|f_{n}(z)-f_{m}(z)\right|+w(z)\left|f_{m}(z)-f(z)\right|$ it follows that for any $\epsilon>0$ there exists $N(\epsilon)$ such that $n, m \geq N$ implies $w(z)\left|f_{n}(z)-f(z)\right|<\frac{1}{2} \epsilon+w(z)\left|f_{m}(z)-f(z)\right|$. We may choose an $m \geq N$, dependent on $z$, such that $w(z)\left|f_{m}(z)-f(z)\right|<\frac{1}{2} \epsilon$. Therefore $n \geq N$ implies $w(z)\left|f_{n}(z)-f(z)\right|<\epsilon$, which implies that $\left\|f_{n}-f\right\| \leq \epsilon$.

Step 4. $f$ is continuous.

We know that for any $\epsilon>0$ there exists $N(\epsilon)$ such that $\left\|f_{n}-f\right\|<\frac{1}{3} \epsilon$ if $n \geq N$. Consequently, $\left|f(z)-f\left(z_{0}\right)\right| \leq\left|f(z)-f_{n}(z)\right|+\left|f_{n}(z)-f_{n}\left(z_{0}\right)\right|+\left|f_{n}\left(z_{0}\right)-f\left(z_{0}\right)\right| \leq \frac{2}{3} \epsilon+\left|f_{N}(z)-f_{N}\left(z_{0}\right)\right|$, since $w(z) \geq 1$. Now the result follows from continuity of $f_{N}$ : for the above $\epsilon$ there exists $\delta(\epsilon)$ such that $\left|z-z_{0}\right|<\delta$ implies $\left|f_{N}(z)-f_{N}\left(z_{0}\right)\right|<\frac{1}{3} \epsilon$.

The only property of $w$ required in the completeness proof is that it have a positive lower bound. This gives one a great deal of flexibility in choosing the Banach space to fit the operator in fixed point or implicit function studies. 


\section{References}

[1] J. Haïssinski, Nuovo Cimento 18 B, 72 (1973)

[2] R. L. Warnock and J. A. Ellison, Proc. 2nd ICFA Advanced Accelerator Workshop on the Physics of High Brightness Beams, UCLA, November 9-12, 1999 (World Scientific, Singapore, 2001); also available as SLAC-PUB-8494.

[3] K. Dressler, Math. Meth. Appl. Sci. 12, 471-487 (1990).

[4] L. Michelotti and F. Mills, Proc. 1989 IEEE Part. Accel. Conf., March 20-23, Chicago, 1801-1803; S. Mane and G. Jackson, ibid., 1394-1396.

[5] K. Yokoya and H. Koiso, Particle Accelerators 27, 181-186 (1990); P. Zenkevich and K. Yokoya, ibid. 40, 229-241 (1997).

[6] A readable introduction to nonlinear functional analysis can be found in T. L. Saaty and J. Bram, Nonlinear Mathematics, (McGraw-Hill, New York, 1964). For many applications of fixed point theory to integral equations see W. Pogorzelski, Integral Equations and Their Applications, Vol.1, (Pergamon, Oxford, 1966). The contraction mapping theorem is as follows: Let $A$ be a transformation of a complete metric space $K$ into itself, $A(K) \subset K$, and suppose that $A$ decreases the distance between elements of $K$ : for a fixed $\beta<1$ we have $d(A(x), A(y)) \leq \beta d(x, y)$, all $x, y \in K$. Then $A$ has a unique fixed point in $K$, which is the limit of the exponentially convergent sequence $x^{(n+1)}=A\left(x^{(n)}\right), x^{(0)} \in K$. Schauder's theorem is a generalization of Brouwer's theorem to the infinite-dimensional case. In a strengthened form due to Mazur (see Pogorzelski, p.415) it is stated as follows: Let $K$ be a closed, convex subset of a Banach space $B$, and $A$ a continuous transformation of $K$ into itself. Then if $A(K)$ is compact, $K$ contains a fixed point of $A$. (By compactness we mean that every infinite subset of $A(K)$ has a limit point in $B$.) Note that uniqueness of the fixed point is not claimed.

[7] R. L. Warnock and J. A. Ellison, Proc. of a Workshop on Beam-Beam Effects in Circular Colliders, Fermilab, June 25-27, 2001, T. Sen and M. Xiao, Eds., available as FERMILAB-Conf-01/390-T.

[8] J. A. Ellison and R. L. Warnock, Proc. 18th Advanced ICFA Beam Dynamics Workshop on Quantum Aspects of Beam Physics, Capri, Italy, October 15-20, 2000 (World Scientific, Singapore, 2002); also available as SLAC-PUB-8778.

[9] S. Y. Lee, Accelerator Physics (World Scientific, Singapore, 2000.)

[10] R. D. Ruth, in Physics of Particle Accelerators, Vol. 1, AIP Conf. Proc. 153, p.150, (Amer. Inst. Phys., New York, 1987).

[11] A. Chao and R. D. Ruth, Particle Accelerators 16, 201-216 (1985).

[12] S. Chandrasekhar, Rev. Mod. Phys 15, 1-91 (1943), reprinted in Selected Papers on Noise and Stochastic Processes, N. Wax, Ed. (Dover, New York, 1954). 
[13] S. G. Krantz and H. R. Parks, The Implicit Function Theorem: History, Theory and Applications (Birkhäuser, Boston, 2002).

[14] J. Dieudonné, Foundations of Modern Analysis (Academic Press, New York, 1969).

[15] T. C. Gard, Introduction to Stochastic Differential Equations (M. Dekker, New York, 1988).

[16] C. W. Gardiner, Handbook of Stochastic Methods (Springer, Berlin, 1996).

[17] N. G. van Kampen, Stochastic Processes in Physics and Chemistry (North Holland, New York, 1981). 